\title{
A 30GHz Wideband CMOS Injection-Locked Frequency Divider for 60GHz Transceiver*
}

\author{
Chunqi Shi, Runxi Zhang, Zongsheng Lai \\ Institute of Microelectronic Circuits and Systems, East China Normal University, Shanghai, China \\ Email: cqshi@ee.ecnu.edu.cn, rxzhang@ee.ecnu.edu.cn
}

Received June, 2013

\begin{abstract}
In this paper, a $30 \mathrm{GHz}$ wide locking-range (26.2 GHz-35.7 GHz) direct injection-locked frequency divider (ILFD), which operating in the millimeter-wave (MMW) band, is presented. The locking range of the ILFD is extended by using differential injection topology. Besides, varactors are used in RLC resonant tank for extending the frequency tuning range. The post simulation results show that a wide locking-range of $9.5 \mathrm{GHz}(30.7 \%)$ is achieved. When the VCO output frequency varies from $26.85 \mathrm{GHz}$ to $34.42 \mathrm{GHz}$, the proposed ILFD can achieve divide-by-two correctly. Designed in $0.13 \mu \mathrm{m}$ CMOS technology, the ILFD occupies a core area of $0.76 \mathrm{~mm}^{2}$ while drawing $7 \mathrm{~mA}$ of current from $2.5 \mathrm{~V}$ power supply.
\end{abstract}

Keywords: CMOS; Injection-locked Frequency Divider (ILFD); Locking Range; VCO; Wideband

\section{Introduction}

With rapid advances in CMOS technology, the CMOS circuit operating in millimeter-wave (MMW) band has attracted increasing interest and research [1-9], such as point-to-point communications, image sensing, and automotive radar systems.

Frequency dividers are key components for frequency synthesizer in a MMW PLL. Conventionally, currentmode-logic (CML) static divider [1], Miller divider [2], and injection-locked frequency divider (ILFD) [3-7] are widely used in various applications. Among these dividers, the CML static diver covers a wide locking range, but its input frequency is low and its power consumption is usually high, compared with ILFDs. For a Miller divider and an ILFD, they have a higher input frequency. However, their locking ranges are quite limited. To realize a divider higher than $20 \mathrm{GHz}$, an ILFD may be one of the good candidates.

This paper describes a differential direct ILFD with varactors for $802.15 .3 \mathrm{c}$ transceiver. Several design considerations for the ILFD are analyzed for increasing the wide locking-range. The paper is organized as follows. Section 2 describes the $60 \mathrm{GHz}$ communication system architecture. Section 3 addresses the analysis and design of an ILFD. Section 4 gives the post simulation result

\footnotetext{
*This paper is funded by project No. 13511500702, Key Laboratory of Wireless Sense Network \& Communication, Shanghai Institute of Microsystem and Information Technology, Chinese Academy of Sciences and ECNU project No. 78210082.
}

and the comparison with some state-of-the-art counterparts. Finally, section 5 gives the conclusion.

\section{System Architecture}

Figure 1 shows a block diagram of the proposed $60 \mathrm{GHz}$ transceiver regulated by $802.15 .3 \mathrm{c}$, in which the $60 \mathrm{GHz}$ LO signal is generated using a frequency double and a 30 GHz PLL. The proposed PLL consists of a phase frequency detector (PFD), charge pump (CP), loop filter, VCO, high speed ILFD as a first divide stage and a series of subsequent frequency CML dividers. According to the system analysis and simulation, the reference frequency is $58.6 \mathrm{MHz}$, the gain of $\mathrm{VCO}$ is $5 \mathrm{GHz} / \mathrm{V}$, the tuning range of $\mathrm{VCO}$ is from $28.5 \mathrm{GHz}$ to $33 \mathrm{GHz}$, and the loop width is $750 \mathrm{kHz}$. The detail design of ILFD will be discussed. The main design challenge is to reduce input capacitance while maintaining a wide operating frequency range.

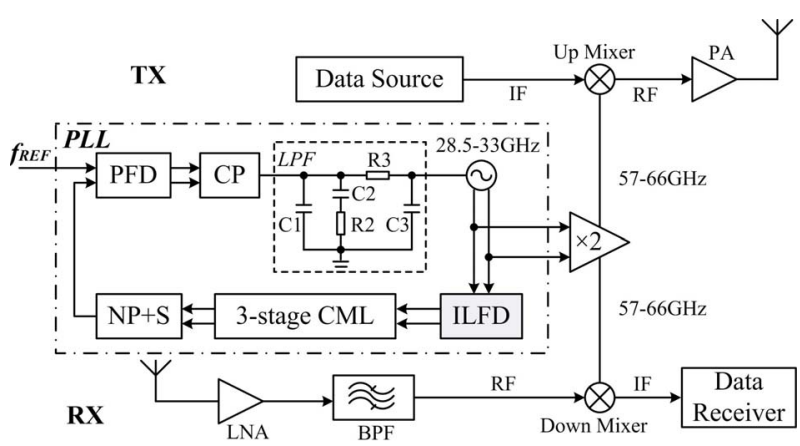

Figure 1. Block diagram of $60 \mathrm{GHz}$ transceiver. 


\section{Circuit Implementation}

A conventional LC-based ILFD is shown in Figure 2(a). The input stage $M_{\text {in }}$ is used to provide both an input signal path and a DC bias path. Thus, $M_{i n}$ is typically large, resulting in a large input capacitance. Moreover, the input signal is significantly degraded by the parasitic capacitor $C_{\text {tail }}$. By using a peaking inductor between the drain terminal of $M_{i n}$ and the ground, this problem can be solved; however, this strategy requires a larger die area. A direct LC-based ILFD is shown in Figure 2(b), it provides a solution for MMW operation with a low input capacitance, but it suffers from a narrow locking range.

\subsection{Circuit Structure}

For wide locking range, the proposed differential ILFD circuit for high-speed operation is shown in Figure 3. The ILFD composed of a conventional LC-VCO, varactors, output buffers, and two transistors (M5, M6) receive the differential injection signals. A complementary crosscoupled pair is used to implement the active $g_{m}$.

\subsection{Locking Range}

In Figure 3, the input transistor M5 and M6 are directly connected to the drain nodes of the differential crosscoupled pair (M1, M2 and M3, M4). An equivalent model of single input transistor is given in Figure 4. (a)

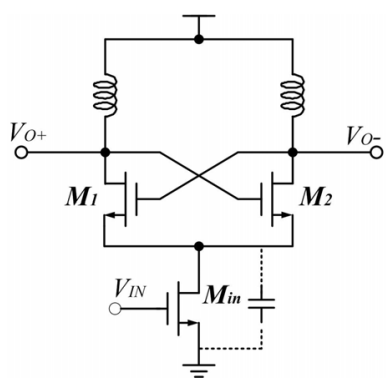

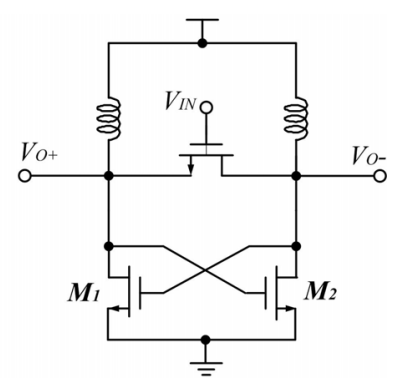

(b)
Figure 2. Conventional ILFD.

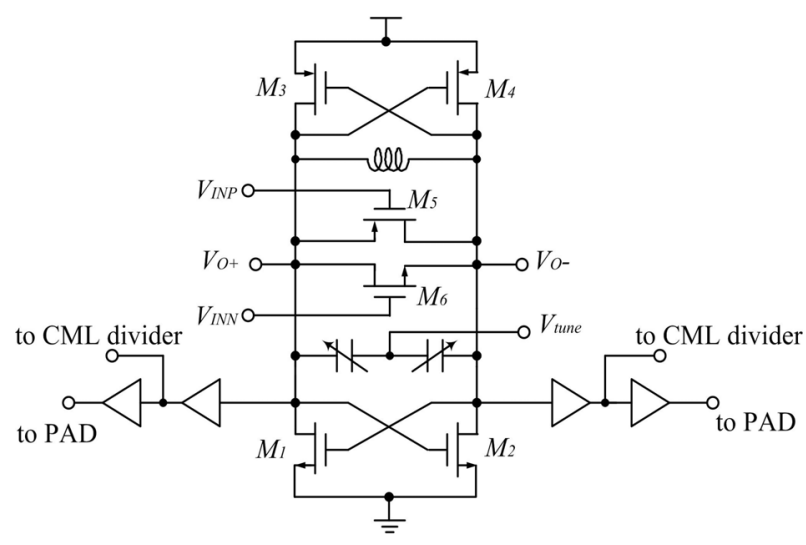

Figure 3. The proposed differential ILFD.

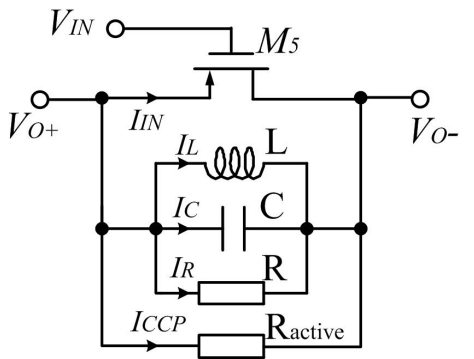

Figure 4. Equivalent model.

$R_{\text {active }}$ is the equivalent resistance of cross-coupled pair; $\mathrm{R}, \mathrm{L}$, and $\mathrm{C}$ represent the equivalent passive load.

Assume that, $V_{\text {in }}(t)=V_{B}+V_{i} \cos (2 \omega t+\theta)$

and

$$
\begin{aligned}
& V_{o-}(t)=V_{C M}-V_{o} \cos \omega t \\
& V_{\text {in }}(t)=V_{B}+V_{i} \cos (2 \omega t+\theta)
\end{aligned}
$$

where $\theta$ stands for the phase difference between the input and the output. The input current $I_{\text {in }}$ can be expressed [8] by separating its in-phase and quadrature phase components as

$$
\begin{aligned}
I_{\text {in }}(t) \approx & {\left[4 K\left(V_{B}-V_{C M}-V_{t h, \text { in }}\right)+2 K V_{i} \cos \theta\right] \cdot V_{o} \cos \omega t } \\
& -\left(2 K V_{i} \sin \theta\right) \cdot V_{o} \sin \omega t
\end{aligned}
$$

where $K=\mu_{n} C_{o x}(W / L)_{i n} / 2$.

$I_{\text {in }}$ in equation (1), can also be expressed by phasor as

$$
\begin{aligned}
& i_{n, 0}=4 K\left(V_{B}-V_{C M}-V_{t h, i n}\right) \cdot V_{o} \cdot e^{j 0^{\circ}}=2 g_{i n, 0} \cdot V_{o} \cdot e^{j 0^{\circ}} \\
& I_{i n, i}=\left(2 K V_{i} \cdot \cos \theta\right) \cdot V_{o} \cdot e^{j 0^{\circ}}=\left(g_{M A X} \cdot \cos \theta\right) \cdot V_{o} \cdot e^{j 0^{\circ}} \\
& I_{i n, q}=-(2 K V \cdot \sin \theta) \cdot V_{o} \cdot e^{j 90^{\circ}}=-\left(g_{M A X} \cdot \sin \theta\right) \cdot V_{o} \cdot e^{j 90^{\circ}}
\end{aligned}
$$

where $g_{i n, 0}$ is the equivalent conductance of the input transistor and $g_{M A X}$ is the equivalent transconductance of the input transistor.

The locking range is derived from two aspects, that is the phase condition and the gain condition.

According the magnitude and phase phasors at node $V_{o^{+}}$shown in Figure 4, the following equality is given:

$$
I_{C}+I_{L}+I_{i n}=0
$$

When the input frequency $\omega_{\text {in }}$ is equal to $2 \omega_{0}$, it result in $I_{C}+I_{L}=0, I_{i n, q}=0$, and $\theta=180^{\circ}$.

When $\omega_{\text {in }}$ is large than $2 \omega_{0}$, the magnitude of $I_{C}$ becomes larger than that of $I_{L}$. The input current should increase the quadrature component $I_{i n, q}$ to compensate it. The corresponding current phasors are shown in Figure 5(a). Similarly, when $\omega_{\text {in }}$ is smaller the $2 \omega_{0}$, the corresponding current phasors are shown in Figure 5(b).

While the magnitude of $I_{i n, q}$ is at its maximum, $\theta$ is equal to $90^{\circ}$ or $270^{\circ}$. The input frequency at this time is defined as the highest locking frequency, $2 \omega_{H}$, and the lowest locking frequency, $2 \omega_{L}$, respectively. The phase 


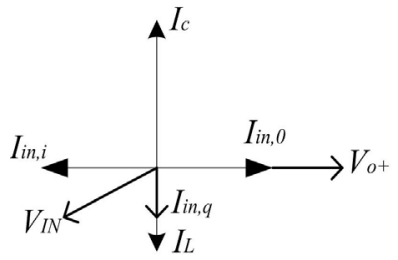

(a) $2 \omega_{0}<\omega_{\text {in }}<2 \omega_{\mathrm{H}}$

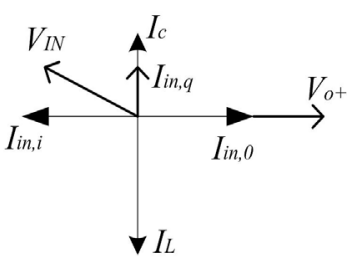

(b) $2 \omega_{\mathrm{L}}<\omega$ in $<2 \omega_{0}$
Figure 5. Current phasors diagram.

shift of the RLC network between $\omega_{0}$ and $\omega_{H}$ or $\omega_{L}$ is expressed as

$$
\tan \phi=\frac{\left|I_{i n, q}\right|}{\left|I_{C C P}\right|} \approx \frac{2 Q_{e f f}}{\omega_{0}}\left(\omega_{0}-\omega_{L}\right), Q_{e f f}=\frac{R_{\mathrm{e} f f}}{\omega_{0} L}
$$

where $R_{\text {eff }}$ is the effective resistance seen by the input transistor. By the phase condition, the locking range (LR) referred to the input is derived as

$$
L R_{\text {phase }}=4\left(\omega_{0}-\omega_{L}\right)=\frac{2 \omega_{0}^{2} L}{\mathrm{R}_{\mathrm{e} f f}} \frac{g_{M A X}}{g_{m}}=\frac{2 g_{M A X}}{g_{m} \mathrm{R}_{\mathrm{e} f f} C}
$$

As for gain condition, if there is no input signal, the loop gain of ILFD should be $\left(g_{m} R_{e f f}\right)^{2}$ and its value should exceed unity to assure oscillating. After injection, the loop gain requirement is given as

$$
\left[\left(g_{m}-g_{M A X} \cos \theta\right) \mathrm{R}_{\mathrm{e} f f}\right]^{2} \geq 1
$$

Also, the gain of the input transistor needs to exceed unity to satisfy the Bark hausen criteria.

The effective impedance of the RLC is expressed as

$$
Z_{\mathrm{tan} k} \approx \frac{R_{\mathrm{e} f f}}{1+j Q_{\text {eff }}(2 \Delta \omega / \omega)}
$$

The locking range determined by the gain condition is derived as

$$
L R_{\text {gain }}=\frac{2 g_{M A X}}{C} \sqrt{1-\left(\frac{1-g_{m} R_{\mathrm{e} f f}}{g_{M A X} R_{\mathrm{e} f f}}\right)^{2}}
$$

If the ILFD is at the edge of oscillation, the locking range is simplified as

$$
L R_{\text {gain }} \approx \frac{2 g_{M A X}}{C}
$$

According to equation (5) and (9), in order to increase the locking range, the capacitance has to be small. Also, $g_{M A X}$ should be large and it is achieved by increasing the size and the input magnitude. The size of cross-coupled pair should be small to realize a smaller $g_{m}$ which will increase the locking range.

In this design, two input transistors, M5 and M6, are used to improve the equivalent transconductance. The simulation results show that the locking range is increase by $50 \%$ compare to single injection topology.

\subsection{Tuning Range}

The locking range of the ILFD is directly related to its tuning range. The output frequency of VCO in this design is from $26.85 \mathrm{GHz}$ to $34.41 \mathrm{GHz}$. For wide tuning range, the varactors are used at the output nodes. The control voltage of ILFD is connected to that of the VCO, which can realize the synchronous tuning. Thus, the tuning range is extended.

\subsection{Output Buffers}

The output buffers in Figure 3 are composed of two stages, the outputs of the first buffer stage are for the next stage divider, and outputs of second buffer stage are for testing. The buffers ensure the stability of the loading capacitance, thus ensure the stability of the tuning frequency.

\section{Simulation Results}

\subsection{Layout and Simulation Results}

The layout of the proposed ILFD is shown in Figure 6. Figure 7 shows the post simulation result of the transient output waveform. When the control voltage $\left(V_{\text {tune }}\right)$ is equal to $2.5 \mathrm{~V}$, the output frequency of VCO is 34.42 $\mathrm{GHz}$, and the output frequency of ILFD is $17.21 \mathrm{GHz}$.

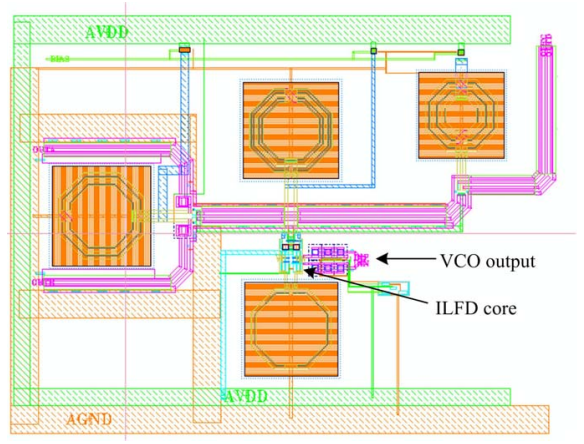

Figure 6. Layout of the propose ILFD.

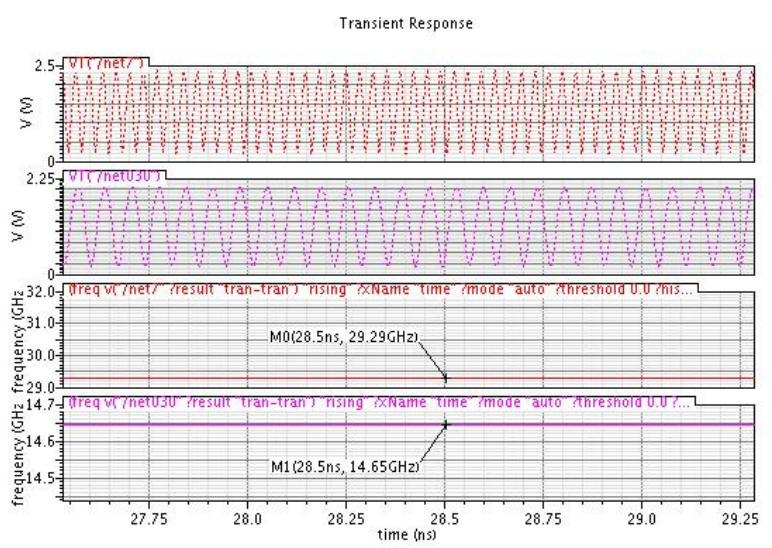

Figure 7. Output waveforms of the proposed ILFD. 


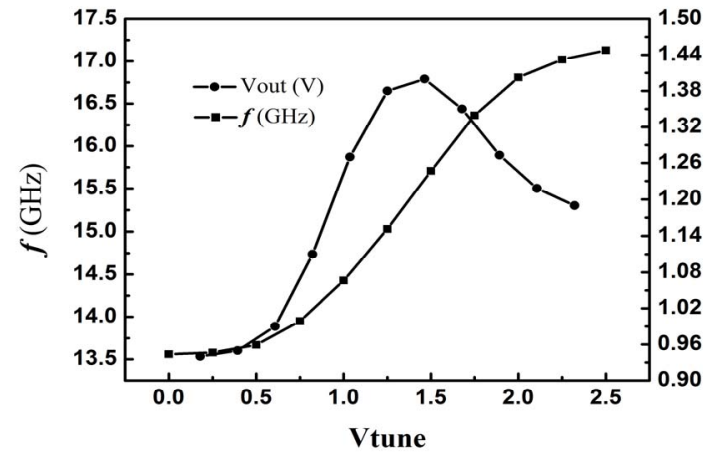

Figure 8. Tuning curves of the ILFD.

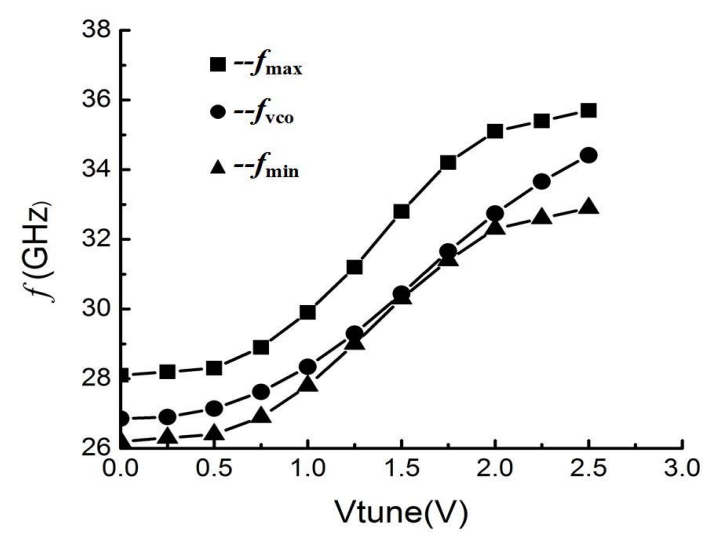

Figure 9. Locking range of the ILFD.

Table 1. Performance summary and comparison.

\begin{tabular}{cccc}
\hline Reference & {$[9]$} & {$[10]$} & This work \\
\hline Process & $0.13 \mu \mathrm{m} \mathrm{CMOS}$ & $0.13 \mu \mathrm{m} \mathrm{CMOS}$ & $0.13 \mu \mathrm{m} \mathrm{CMOS}$ \\
Divided number & 2 & 2 & 2 \\
VDD & $1 \mathrm{~V}$ & $0.8 \mathrm{~V}$ & $2.5 \mathrm{~V}$ \\
$\begin{array}{c}\text { Input } \\
\text { frequency }\end{array}$ & $70 \mathrm{GHz}$ & $63 \mathrm{GHz}$ & $30 \mathrm{GHz}$ \\
$\begin{array}{c}\text { Locking Range } \\
\begin{array}{c}\text { With/Without } \\
\text { varactors }\end{array}\end{array}$ & $13.57 \%$ & $11.7 \%$ & $30.7 \%$ \\
$\begin{array}{c}\text { Input Power } \\
\text { Power }\end{array}$ & $5 \mathrm{dBm}$ & $0 \mathrm{dBm}$ & $7 \mathrm{dBm}$ \\
consumption & $4.4 \mathrm{~mW}$ & $1.6 \mathrm{~mW}$ & $17.5 \mathrm{~mW}$ \\
\hline
\end{tabular}

Figure 8 shows the tuning curves of the ILFD, the tuning frequency varies from $13.56 \mathrm{GHz}$ to $17.13 \mathrm{GHz}$ when the $V_{\text {tune }}$ varies from 0 to $2.5 \mathrm{~V}$. The locking range of ILFD is shown in Figure 9, the middle curve is the VCO output frequency, and this curve is always between the minimum and maximum tuning range of ILFD. The ILFD realizes the correct divide-by-two function when the VCO output frequency varies from $26.85 \mathrm{GHz}$ to
$34.42 \mathrm{GHz}$, and the locking range is $9.5 \mathrm{GHz}(30.7 \%)$

\subsection{Performance Comparison}

The post simulation results of the proposed ILFD are summarized in Table 1, also the comparison with the reported state-of-the-art ILFD realized in $0.13 \mu \mathrm{m}$ CMOS process is given. According to the comparison results, the propose ILFD has a larger locking range, but consumes more power.

\section{Conclusions}

To meet the requirement of the $60 \mathrm{GHz}$ transceiver, a wide locking range ILFD is presented in this paper. The locking ranges of the ILFD are analyzed from the phase and gain conditions, respectively. Based on the analysis, a $30 \mathrm{GHz}$ direct differential ILFD has been designed in $0.13 \mu \mathrm{m}$ CMOS technology. The post simulation results show that the locking range of the proposed ILFD is from $26.2 \mathrm{GHz}$ to $35.7 \mathrm{GHz}$ (30.7\%), which satisfies the bandwidth requirement of IEEE 802.15.3c protocol.

\section{REFERENCES}

[1] C. Lee and S. I. Liu, "A 58-to-60.4 GHz Frequency Synthesizer in $90 \mathrm{~nm}$ CMOS," in IEEE Int. Solid-State Circuits Conf. (ISSCC) Dig. Tech. Papers, 2007, pp. 196-197.

[2] J. Lee and B. Razavi, "A 40-GHz Frequency Divider in 0.18- $\mu$ m CMOS Technology," IEEE J. Solid-State Circuits, Vol. 39, No. 4, pp. 594-601, Apr. 2004. doi:10.1109/JSSC.2004.825119

[3] P. Mayr, C. Weyers and U. Langmann, "A $90 \mathrm{GHz} 65 \mathrm{~nm}$ CMOS Injection-locked Frequency Divider," in IEEE Int. Solid-State Circuits Conf. (ISSCC) Dig. Tech. Papers, Feb. 2007, pp. 198-199.

[4] K. H. Tsai, L. C. Cho, J. H. Wu and S. I. Liu, "3.5 mW W-band Frequency Divider with Wide Locking Lange in 90 nm CMOS Technology," IEEE Int. Solid-State Circuits Conf. (ISSCC) Dig. Tech. Papers, 2008, pp. 466-467.

[5] L. C. Cho, K. H. Tsai, C. C. Hung and S. I. Liu, "93.5-109.4 GHz CMOS Injection-locked Frequency Divider with 15.3\% Locking Range," Symp. VLSI Circuits Dig. Tech. Papers, 2008, pp. 266-267.

[6] B. Y. Lin, K. H. Tsai and S. I. Liu, "A 128.24- to- 137.00 $\mathrm{GHz}$ Injection Locked Frequency Divider In $65 \mathrm{~nm}$ CMOS," IEEE Int. Solid-State Circuits Conf. (ISSCC) Dig. Tech. Papers, 2009, pp. 282-283.

[7] B. Y. Lin and S. I. Liu, "A 132.7-to-143.5 GHz Injection-locked Frequency Divider in 65 nm CMOS," Symp. VLSI Circuits Dig. Tech. Papers, 2009, pp. 230-231.

[8] B. Y. Lin and S. I. Liu, "Analysis and Design of D-band Injection-locked Frequency Dividers," IEEE J. Solid-State Circuits, Vol. 46, No. 6, pp. 1250-1264, June 2011. doi:10.1109/JSSC.2011.2131750 
[9] C. Y. Wu and C. Y. Yu, "Design and Analysis of Millimeter-wave Direct Injection-locked Frequency Divider with Large Frequency Locking Range," IEEE Transactions on Microwave Theory and Techniques, Vol. 55, No. 8, 2007, pp. 1649-1658. doi:10.1109/TMTT.2007.902067
[10] S. J. Rong, W. L. Alan and H. C. Luong, " $0.9 \mathrm{~mW} 7 \mathrm{GHz}$ and $1.6 \mathrm{~mW} 60 \mathrm{GHz}$ Frequency Dividers with Locking-range Enhancement in $0.13 \mu \mathrm{m}$ CMOS," IEEE Int. Solid-State Circuits Conf. (ISSCC) Dig. Tech. Papers, 2009, pp. 96-97. 